\title{
Incidencia de la Coordinación en el Golpe de Balón de Borde Interno en las Deportistas de la Categoría Sub 18
}

\author{
Orlando David Mazón Moreno, \\ Hugo Santiago Trujillo Chávez,
}

Magíster en Cultura Física y Entrenamiento Deportivo

Docente de la Escuela Superior Politécnica de Chimborazo

Danilo Ortiz Fernández,

Magíster en Actividad Física

Docente de la Escuela Superior Politécnica de Chimborazo, Ecuador

Dennys Germán Palacios Valdiviezo,

Licenciado en Cultura Física y Entrenamiento Deportivo

Técnico Docente de la Escuela Superior Politécnica de Chimborazo, Ecuador

Usca Moreno Renán Patricio,

Licenciado en Cultura Física y Entrenamiento Deportivo

Doi: 10.19044/esj.2019.v15n3p239 URL:http://dx.doi.org/10.19044/esj.2019.v15n3p239

\section{Resumen}

El objetivo de la investigación fue determinar la incidencia de un programa para mejorar la coordinación del golpe de balón con borde interno en las deportistas de la categoría sub 18 de la Academia de Fútbol Femenino Innovagol de la ciudad de Riobamba en el año 2016. La investigación fue cuasi experimental, de campo, bibliográfica hipotética deductiva. Se consideró una población y muestra no intencionada de 50 deportistas de sexo femenino, quienes fueron encuestadas a fin de obtener una línea base que permitió elaborar un programa de intervención con ejercicios diarios de mejoramiento de coordinación y tuvo una duración de 6 semanas. Los datos obtenidos de las encuestas fueron tabulados, analizados e interpretados. Posteriormente las deportistas fueron evaluadas mediante un test de coordinación (escalera) que ha sido utilizado y validado en investigaciones anteriores. La encuesta se realizó en dos momentos (Pre y Post test). Los resultados obtenidos fueron procesados y sometidos a una prueba estadística que permitió establecer que los resultados no fueron significativos y que sólo la coordinación no es suficiente para incidir positivamente en el golpe del balón con borde interno, por lo cual es necesario considerar también la precisión en el golpe del balón. 
Palabras claves: Coordinación, golpe balón, borde interno, incidencia, deportistas femeninas

\section{Incidence of Coordination in Shooting the Ball with Inner Curve by the Athletes of the Sub 18 Category}

\section{Orlando David Mazón Moreno,} Hugo Santiago Trujillo Chávez,

Magíster en Cultura Física y Entrenamiento Deportivo

Docente de la Escuela Superior Politécnica de Chimborazo

Danilo Ortiz Fernández,

Magíster en Actividad Física

Docente de la Escuela Superior Politécnica de Chimborazo, Ecuador

\section{Dennys Germán Palacios Valdiviezo,}

Licenciado en Cultura Física y Entrenamiento Deportivo

Técnico Docente de la Escuela Superior Politécnica de Chimborazo, Ecuador

Usca Moreno Renán Patricio,

Licenciado en Cultura Física y Entrenamiento Deportivo 
Keywords: Coordination, Ball blow, Inner Curve, Incidence, Female Athletes

\section{Introducción}

Desde el punto de vista deportivo, la coordinación es importante porque, tal como señala Weineck (1988), "permite al deportista dominar las acciones motoras con precisión y economía, en situaciones determinadas que pueden ser previstas o imprevistas".

Torres (2005) menciona que la coordinación "es la capacidad del organismo para ejecutar una acción motriz controlada, con precisión y eficacia". Las capacidades coordinativas generales o básicas son aquellas que incluyen la precisión o regulación y la dirección del movimiento y se dividen en:

- Capacidad reguladora del movimiento; que según Ruiz (s/a), se define como fundamental debido a que sería imposible desarrollar las demás sin la regulación del movimiento.

- Capacidad de adaptación y cambio motrices, que según Lorenzo (2002), es la capacidad de cambio para adaptar el programa de acción motora a las nuevas situaciones, en base a los cambios situativos percibidos o anticipados durante la ejecución motora o la prosecución de la acción en forma completamente diferente. Esta capacidad se desarrolla cuando el organismo es capaza de adaptarse a las condiciones de los movimientos cuando se presente una nueva situación y tiene que cambiar y volver a adaptarse. En otras palabras se define, como la capacidad que tiene el organismo de adaptarse a las diferentes situaciones y condiciones en que se realizan movimientos.

En lo que respecta a coordinación como tal, Weineck (2005) afirma que la capacidad coordinativa de orientación permite determinar y modificar la situación y los movimientos del cuerpo en el espacio y en el tiempo, en relación con campo de acción definido y/o con un objeto en movimiento.

Schreinner (2005) sostiene de igual forma que "la coordinación es la acción combinada del sistema nervioso central y de la musculatura esquelética encaminada al desarrollo de un movimiento planificado."

López y Garoz (2004) clasifican la coordinación como variada, pero hoy en día se reconocen dos grandes grupos: la coordinación dinámica general que regula los movimientos corporales globales y que es la base de las habilidades motrices, junto al equilibrio y la coordinación óculo segmentaria que implica un ajuste de la vista y el segmento corporal.

Otras clasificaciones actuales y específicas no tan genéricas son las que propone Torres (2005), el cual agrupa la coordinación en diferentes ramas: 
- Coordinación Gruesa: aquella que es necesaria para realizar movimientos amplios y generales.

- Coordinación Fina: Es aquella coordinación que necesita más precisión por la delicadeza de sus movimientos.

- Coordinación Segmentaria: Se refiere a la coordinación que se requiere de una parte de nuestro cuerpo.

- Coordinación General: Regula los movimientos globales.

- Coordinación Óculo-Manual: es la que se emplea por ejemplo en baloncesto, donde llevamos un móvil en las manos.

- Coordinación Óculo-Pédica: es aquella que se requiere en el fútbol, donde el móvil es llevado en los pies.

Para la evaluación de la coordinación no es fácil establecer test o pruebas útiles para medirla y evaluarla porque esta habilidad está unida a otras capacidades. Aun así, se pueden establecer algunos test como el examen psicomotor de Mazzo y de Vayer y la observación psicomotriz de Da Fonseca (López, 2004).

El entrenamiento coordinativo específico de un tipo de deporte es un entrenamiento de aplicación de movimientos aprendidos y bajo condiciones (de dificultad) especiales. El entrenamiento de fútbol se trata de un aprendizaje rápido y efectivo de las técnicas de fútbol que sean necesarias. En conexión con ello, las capacidades coordinativas juegan un papel muy importante.

En lo referido al fútbol, la coordinación debe ser sometida a entrenamiento, de modo que el jugador pueda dominar los desarrollos de movimientos específicos y pueda ajustar los factores de perturbación propios de su tipo de deporte. Cuanto más elevado sea el nivel de rendimiento del futbolista, más variados deben ser los contenidos del entrenamiento de coordinación, desde los puntos esenciales generales hasta los específicos del fútbol. Las técnicas futbolísticas van ganando en importancia, mientras que los ejercicios para la mejora de las capacidades de coordinación generales se van quedando en un segundo plano.

En lo que respecta a las escuelas de fútbol de Ecuador se han encontrado factores negativos tales como: falta de entrenadores deportivos, tener una visión competitiva y no más formativa integral, conceptualizar al deportista como una mujer en miniatura y no como mujer en potencia; ya que han trasladado las formas de enseñanza de los adultos hacia los niños desde su experiencia empírica, sin establecer bases que sirvan para la continua progresión o como referencia para futuros jugadores. Otro facor negativo relevante es la falta de conocimiento en el aspecto sociológico de la deportista; se debe recordar que la misión como entes de influencia es llevar al infante a amar al deporte y formar el hábito del practicante deportivo. 
Ante esta realidad latente, la causa principal de la investigación fue dar a conocer que se pueden corregir estas falencias tomando en cuenta que la formación de la deportista en el fútbol es muy amplia y que existen áreas indispensables que son pilares de un jugador de calidad como la coordinación y sus capacidades coordinativas, siendo esta área la parte vertebral de la técnica deportiva.

Durante décadas, los entrenadores fijaron su atención en las capacidades condicionales: la fuerza, la resistencia y la velocidad. Fue justamente con el pasar del tiempo y frente a un escenario cada vez más competitivo, que ampliaron su horizonte hacia otras capacidades íntimamente relacionadas con el sistema nervioso. Son dichas s cualidades, conocidas hoy como capacidades coordinativas, las que permiten al deportista realizar los movimientos con precisión, economía y eficacia.

\section{Metodología}

La presente investigación constituye un paso importante en la metodología, pues va a determinar el enfoque y el mejoramiento de la misma. Por tal motivo, se trabajó con un enfoque cuantitativo hacia la coordinación en las deportistas de la categoría sub 18 de la Academia de Fútbol Femenino Innovagol de la ciudad de Riobamba.

La investigación fue bibliográfica y documental, pues se revisaron, analizaron, ampliaron, profundizaron y compararon diferentes puntos de vista de varios autores, teorías, criterios y temas referentes en el desarrollo de la coordinación en el golpe de balón de borde interno. La investigación también es de campo pues se ejecutó en el lugar de los hechos, con las estudiantes de la Academia de Fútbol Femenino Innovagol de la ciudad de Riobamba.

En lo que respecta a la población, se consideró para la muestra a 50 deportistas de sexo femenino de la categoría sub 18 estudiante de la academia antes mencionada en el año 2016, grupo que representa a toda la población; es decir, es una muestra no intencionada.

La investigación inició con la aplicación de un cuestionario cerrado con el fin de levantar una línea base de la variable independiente representada por la coordinación para incidir en el golpe de balón de borde interno. La información obtenida fue tabulada, analizada e interpretada a través de tablas y gráfico de pastel, información que permitió ratificar y establecer un plan de intervención de ejercicio de coordinación, el mismo que contempló un test de coordinación para incidir en el golpe de balón de borde interno. El plan de intervención consistió en un entrenamiento diario que fue planificado en un periodo de 6 semanas y durante el cual se utilizó el test que permitió visualizar todos los aspectos relacionados con la investigación realizada. Para la recolección de la información se utilizó un test de la escalera. 
Los datos obtenidos fueron evaluados en dos momentos: antes y después de aplicar el plan de intervención. Dichos datos fueron confrontados con una prueba estadística que permitió determinar la incidencia de la coordinación en el golpe de balón de borde interno.

\section{Resultados}

Análisis e interpretación de resultados de encuestas

Pregunta 1. ¿Sabe usted qué es la coordinación?

Tabla 1. Conocimiento de la coordinación

\begin{tabular}{|c|c|c|}
\hline Alternativa & Frecuencia & Porcentaje \\
\hline Sí & 19 & $38 \%$ \\
\hline No & 31 & $62 \%$ \\
\hline TOTAL & 50 & $100 \%$ \\
\hline
\end{tabular}

Fuente: Encuesta a deportistas

Elaborado por: Usca Renán, 2016

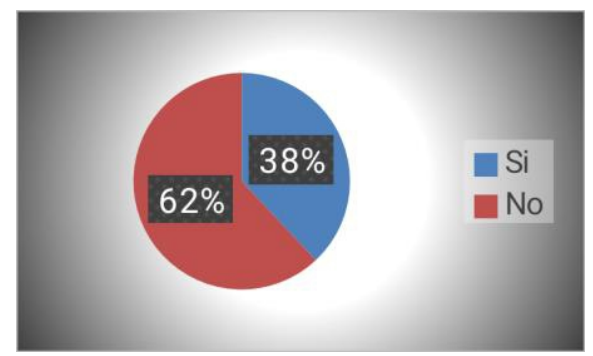

Gráfico 1. Conocimiento de la coordinación

Análisis: Mediante los cuadros estadísticos es posible ver que el $38 \%$ de deportistas encuestadas dicen que sí conocen qué es la coordinación, mientras que el $62 \%$ dicen que no conocen esto.

Interpretación: De acuerdo al análisis gráfico se ve que la mayoría de las deportistas de la Academia de Fútbol Femenino Innovagol desconocen qué es la coordinación por lo que se debe trabajar en ese aspecto para que ellas sepan la importancia de la misma.

Pregunta 2. ¿Usted cree que para golpear el balón con borde interno es necesario tener coordinación?

Tabla 2. Conocimiento de golpe con borde interno con coordinación

\begin{tabular}{|c|c|c|}
\hline Alternativa & Frecuencia & Porcentaje \\
\hline Sí & 17 & $34 \%$ \\
No & 33 & $66 \%$ \\
TOTAL & 50 & $100 \%$ \\
\hline
\end{tabular}

Fuente: Encuesta a deportistas

Elaborado por: Usca Renán, 2016

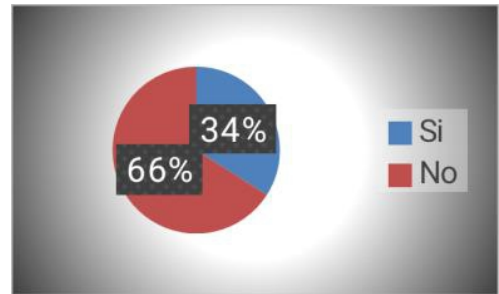

Gráfico 2. Coordinación para golpear el balón

Análisis: Los cuadros estadísticos muestran que el $34 \%$ de las deportistas encuestadas dicen que sí es necesario tener coordinación para golpear el balón con borde interno, mientras que el $66 \%$ dicen que no lo es o por lo menos no conocen del tema. 
Interpretación: De acuerdo al análisis gráfico se ve que la mayoría de las deportistas de la academia de fútbol femenino Innovagol desconocen sobre este tema por lo que es importante trabajar en ese aspecto para que las deportistas estén al tanto de la necesidad de tener una buena coordinación previo al golpe de balón borde interno.

Pregunta 3.- ¿Su entrenador con qué frecuencia realiza ejercicios de coordinación en los entrenamientos?

Tabla 3. Entrenamiento de la coordinación

\begin{tabular}{|c|c|c|}
\hline Alternativa & Frecuencia & Porcentaje \\
\hline 1 vez a la semana & 27 & $54 \%$ \\
2 veces a la semana & 8 & $16 \%$ \\
3 veces por semana & 4 & $8 \%$ \\
Nunca & 11 & $22 \%$ \\
\hline TOTAL & 50 & $100 \%$ \\
\hline
\end{tabular}

Fuente: Encuesta a deportistas

Elaborado por: Usca Renán, 2016

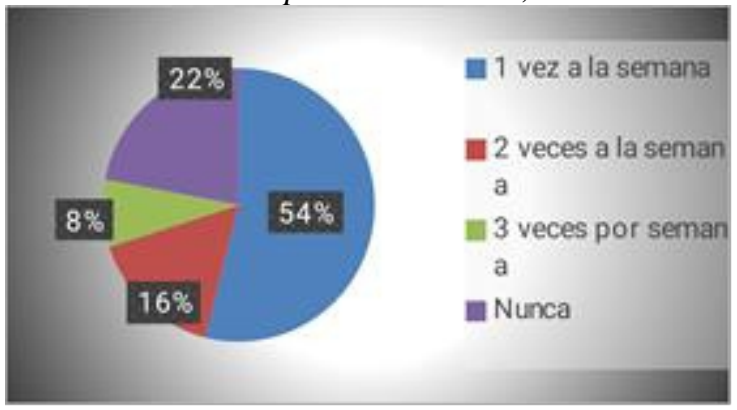

Gráfico 3. Entrenamiento de la coordinación

Análisis: Los cuadros estadísticos muestran que el $54 \%$ de deportistas encuestadas dicen que $1 \mathrm{vez}$ a la semana, el $16 \%$ de deportistas encuestadas dicen que 2 veces a la semana, el $8 \%$ dice que 3 veces a la semana, y el $22 \%$ de deportistas encuestadas dicen que nunca realiza ejercicios de coordinación en los entrenamientos.

Interpretación: De acuerdo al análisis gráfico se ve que la mayoría de las deportistas de la Academia de Fútbol Femenino Innovagol no realizan este tipo de entrenamiento como es la coordinación, por lo cual es importante trabajar en este aspecto para que las deportistas logren alcanzar el nivel de trabajo esperado por parte de sus entrenadores. 
Pregunta 4.- ¿Cree usted que es importante entrenar la coordinación?

Tabla 4. Entrenar la coordinación

\begin{tabular}{|c|c|c|}
\hline Alternativa & Frecuencia & Porcentaje \\
\hline $\mathrm{Si}$ & 20 & $40 \%$ \\
$\mathrm{No}$ & 30 & $60 \%$ \\
\hline $\mathrm{TOTAL}$ & 50 & $100 \%$ \\
\hline
\end{tabular}

Fuente: Encuesta a deportistas

Elaborado por: Usca Renán, 2016

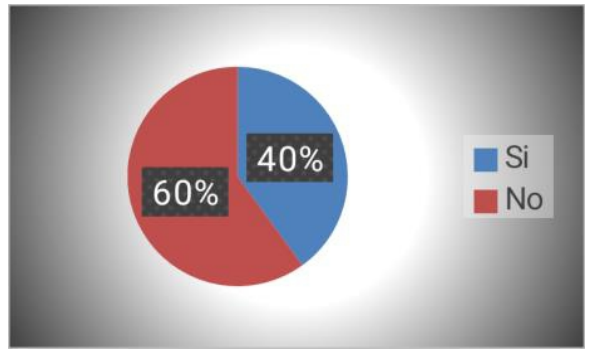

Gráfico 4. Entrenar la coordinación

Análisis: Mediante los cuadros estadísticos se puede observar que el $40 \%$ de deportistas encuestadas dicen que sí es importante entrenar la coordinación, mientras que el $60 \%$ dicen que no es importante o por lo menos no sabían que es de suma importancia.

Interpretación: De acuerdo al análisis gráfico se ve que la mayoría de las deportistas de la Academia de Fútbol Femenino Innovagol desconocen sobre este tema por lo que se debe trabajar en ese aspecto tanto deportivo como motivacional para que las deportistas conozcan lo importante que es entrenar la coordinación.

Pregunta 5. ¿Su entrenador le ha evaluado la coordinación mediante algún test?

Tabla 5. Evalúan su coordinación

\begin{tabular}{|c|c|c|}
\hline Alternativa & Frecuencia & Porcentaje \\
\hline $\mathrm{Si}$ & 15 & $30 \%$ \\
$\mathrm{No}$ & 35 & $70 \%$ \\
\hline TOTAL & 50 & $100 \%$ \\
\hline
\end{tabular}

Fuente: Encuesta a deportistas

Elaborado por: Usca Renán, 2016

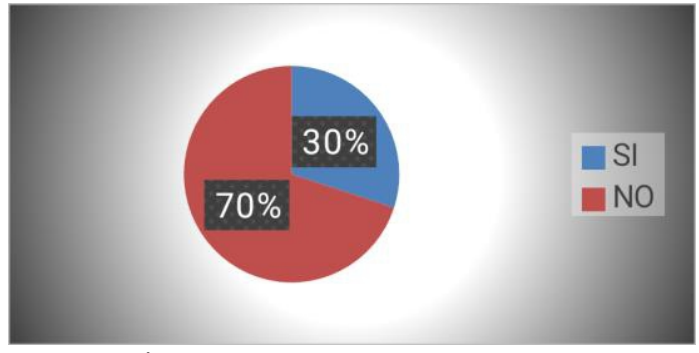

Gráfico 5. Evalúan su coordinación

Análisis: A partir de los cuadros estadísticos es posible observar que el $30 \%$ de deportistas encuestadas dicen que sí han sido evaluadas, mientras que el 70 $\%$ dice que no han sido evaluadas.

Interpretación: De acuerdo al análisis gráfico se ve que la mayoría de las deportistas de la Academia de Fútbol Femenino Innovagol no han sido evaluadas mediante un test de coordinación. 
Pregunta 6.- ¿Cuándo usted golpea el balón con el borde interno, el balón se dirige donde usted desea?

Tabla 6. Dirección del balón al golpearlo

\begin{tabular}{|c|c|c|}
\hline Alternativa & Frecuencia & Porcentaje \\
\hline $\mathrm{Si}$ & 12 & $24 \%$ \\
$\mathrm{No}$ & 38 & $76 \%$ \\
\hline TOTAL & 50 & $100 \%$ \\
\hline
\end{tabular}

Fuente: Encuesta a deportistas

Elaborado por: Usca Renán, 2016

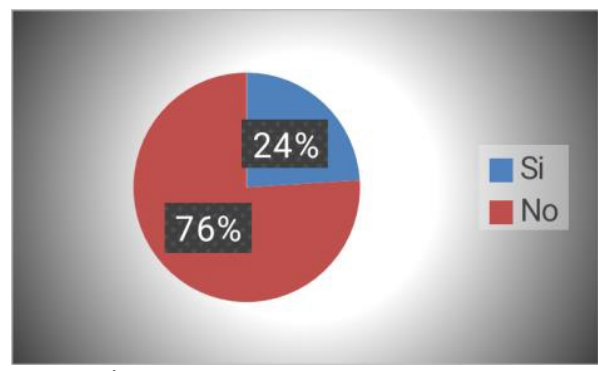

Gráfico 6. Dirección del balón al golpearlo

Análisis: Los cuadros estadísticos muestran que el $76 \%$ de deportistas encuestadas dicen que cuando golpean el balón con borde interno, el balón no se dirige al destino deseado, mientras que el $24 \%$ dice que cuando golpean el balón con borde interno, el balón si se dirige al destino deseado.

Interpretación: De acuerdo al análisis gráfico se puede afirmar que el balón no se dirige al destino deseado cuando la mayoría de las deportistas de la Academia de Fútbol Femenino Innovagol golpea el balón con borde interno.

Pregunta 7. ¿Para tener precisión en el golpe del balón con el borde interno qué piensa que debería entrenarse?

Tabla 7. Lo que debe entrenarse

\begin{tabular}{|l|c|c|}
\hline Alternativa & Frecuencia & Porcentaje \\
\hline Resistencia & 0 & $0 \%$ \\
Fuerza & 24 & $48 \%$ \\
Velocidad & 17 & $34 \%$ \\
Coordinación & 9 & $18 \%$ \\
\hline TOTAL & $\mathbf{5 0}$ & $\mathbf{1 0 0 \%}$ \\
\hline
\end{tabular}

Fuente: Encuesta a deportistas

Elaborado por: Usca Renán, 2016

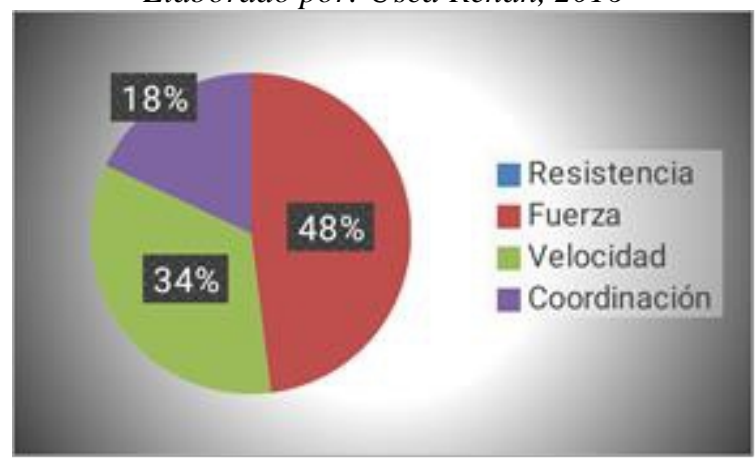

Gráfico 7. Lo que debe entrenarse 
Análisis: A partir de los cuadros estadísticos se puede observar que el $48 \%$ de deportistas encuestadas dicen que la fuerza debería entrenarse, el $34 \%$ de deportistas encuestadas dicen que la velocidad debería entrenarse, y el $18 \%$ de deportistas encuestadas dicen la coordinación.

Interpretación: De acuerdo al análisis grafico se ve que la mayoría de las deportistas de la Academia de Fútbol Femenino Innovagol desconocen del tema. Por esta razón se debe trabajar en ese aspecto para que las deportistas sepan que para tener precisión en el golpe del balón con el borde debería entrenarse la coordinación.

Pregunta 8.- ¿Cree usted que su entrenador debería disponer de un programa de ejercicios para mejorar la coordinación en la precisión del balón con el borde interno?

Tabla 8. Disponer de un programa de ejercicio de coordinación

\begin{tabular}{|l|c|c|}
\hline Alternativa & Frecuencia & Porcentaje \\
\hline $\mathrm{Si}$ & 45 & $90 \%$ \\
$\mathrm{No}$ & 5 & $10 \%$ \\
\hline TOTAL & 50 & $100 \%$ \\
\hline
\end{tabular}

Fuente: Encuesta a deportistas

Elaborado por: Usca Renán, 2016

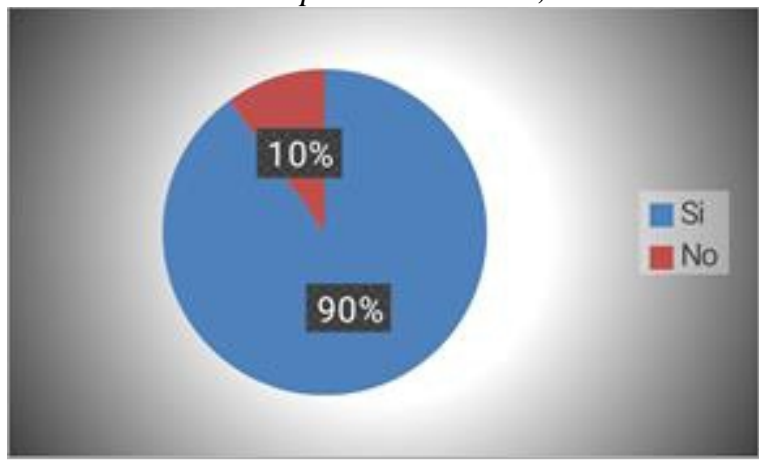

Gráfica 8. Disponer de un programa de ejercicio de coordinación

Análisis: Mediante los cuadros estadísticos es posible observar que el $90 \%$ de las deportistas encuestadas dicen que su entrenador debería disponer de un programa de ejercicios para mejorar la coordinación en la precisión del balón con el borde interno, mientras que el $10 \%$ dicen que su entrenador no debería disponer de un programa de ejercicios para mejorar la coordinación en la precisión del balón con el borde interno.

Interpretación: De acuerdo al análisis gráfico se puede notar que la mayoría de deportistas de la Academia de Fútbol Femenino Innovagol dice que su entrenador debería disponer de un programa de ejercicios para mejorar la coordinación en la precisión del balón con el borde interno, por lo que este se debería emplear en su plan de entrenamiento. 
Tabla 9. Análisis comparativo de pre y post test de coordinación

\begin{tabular}{|c|c|c|c|c|c|c|c|c|c|}
\hline \multirow{2}{*}{ Deportista } & \multicolumn{2}{|c|}{ Pre test } & \multicolumn{2}{|c|}{ Post test } & \multirow{2}{*}{ Deportista } & \multicolumn{2}{|c|}{ Pre test } & \multicolumn{2}{|c|}{ Post test } \\
\hline & Tiempo & Categoria & Tiempo & Categoria & & Tiempo & Categoria & Tiempo & Categoria \\
\hline 1 & 3”32 & Excelente & $3 " 30$ & Excelente & 26 & 4"98 & Buena & 4"99 & Buena \\
\hline \multirow{2}{*}{2} & \multirow{2}{*}{$4 ” 20$} & \multirow{2}{*}{$\begin{array}{c}\text { Muy } \\
\text { buena }\end{array}$} & \multirow{2}{*}{$4 " 15$} & \multirow{2}{*}{$\begin{array}{c}\text { Muy } \\
\text { buena }\end{array}$} & 27 & $5 " 80$ & Regular & $5 ” 77$ & Regular \\
\hline & & & & & 28 & $5 " 02$ & Buena & $4 " 97$ & Buena \\
\hline 3 & $5 " 50$ & Regular & $5 " 47$ & Regular & \multirow[b]{2}{*}{29} & \multirow{2}{*}{$4 ” 25$} & \multirow{2}{*}{$\begin{array}{c}\text { Muy } \\
\text { buena }\end{array}$} & \multirow{2}{*}{ 4”22 } & \multirow{2}{*}{ Muy buena } \\
\hline 4 & $5 " 61$ & Regular & $5 " 50$ & Regular & & & & & \\
\hline \multirow{2}{*}{5} & \multirow{2}{*}{$4 " 60$} & \multirow{2}{*}{ Buena } & \multirow{2}{*}{$4 " 52$} & \multirow{2}{*}{$\begin{array}{c}\text { Muy } \\
\text { buena }\end{array}$} & 30 & $5 " 90$ & Regular & $5 " 93$ & Regular \\
\hline & & & & & 31 & 4"11 & Excelente & 4"07 & Excelente \\
\hline 6 & $5 " 89$ & Regular & $5 " 80$ & Regular & 32 & $5 " 10$ & Buena & 5"06 & Buena \\
\hline 7 & 3"96 & Excelente & $3 " 92$ & Excelente & 33 & $5 " 45$ & Buena & $5 " 46$ & Buena \\
\hline 8 & $5 " 51$ & Regular & $5 " 47$ & Regular & 34 & $5 " 73$ & Regular & $5 " 71$ & Regular \\
\hline 9 & 6"12 & Deficiente & $5 " 99$ & Deficiente & \multirow{2}{*}{35} & \multirow{2}{*}{ 4"15 } & \multirow{2}{*}{$\begin{array}{c}\text { Muy } \\
\text { buena }\end{array}$} & \multirow{2}{*}{ 4"11 } & \multirow{2}{*}{ Excelente } \\
\hline 10 & $5 ” 06$ & Buena & $5 " 02$ & Buena & & & & & \\
\hline 11 & 4"91 & Buena & 4"87 & Buena & 36 & $5 " 82$ & Regular & $5 " 81$ & Regular \\
\hline 12 & 4"02 & Excelente & $4 " 02$ & Excelente & 37 & $5 " 79$ & Regular & $5 " 74$ & Regular \\
\hline 13 & $5 " 52$ & Regular & $5 " 49$ & Regular & \multirow{2}{*}{38} & \multirow{2}{*}{ 4"45 } & Muy & $4 " 44$ & Muy buena \\
\hline 14 & $5 " 58$ & Regular & $5 " 57$ & Regular & & & buena & 4,44 & Muy buena \\
\hline 15 & $6 " 00$ & Deficiente & $5 " 98$ & Deficiente & 39 & $5 " 48$ & Regular & $5 " 50$ & Regular \\
\hline 16 & $4 " 73$ & Buena & $4 " 70$ & Buena & 40 & 4"14 & Excelente & 4"11 & Excelente \\
\hline 17 & $4 " 55$ & Muy & $4 " 57$ & Muy & 41 & $5 " 55$ & Regular & $5 " 51$ & Regular \\
\hline 17 & & buena & & buena & 42 & $5 " 66$ & Regular & $5 " 67$ & Regular \\
\hline 18 & 4"86 & Buena & 4"85 & Buena & 43 & $5 " 09$ & Buena & $5 " 01$ & Buena \\
\hline & & Muy & & Muy & 44 & $5 " 70$ & Regular & $5 " 69$ & Regular \\
\hline 19 & $4 " 17$ & buena & $4 " 15$ & buena & 45 & $5 " 85$ & Regular & $5 " 82$ & Regular \\
\hline 20 & $5 " 64$ & Regular & $5 " 62$ & Regular & 46 & $5 " 98$ & Deficiente & $5 " 95$ & Deficiente \\
\hline 21 & $5 " 42$ & Buena & $5 " 37$ & Buena & 47 & $5 " 23$ & Buena & $5 " 25$ & Buena \\
\hline 22 & $5 " 74$ & Regular & $5 " 71$ & Regular & & & Muy & & \\
\hline 23 & $5 " 30$ & Buena & $5 " 32$ & Buena & 48 & 4"51 & buena & $4 " 48$ & Muy buena \\
\hline 24 & $4 " 34$ & Muy & $4 " 30$ & Muy & 49 & $6 ” 21$ & Deficiente & $6 ” 20$ & Deficiente \\
\hline 24 & 434 & buena & 430 & buena & 50 & 6"01 & Deficiente & $5 " 99$ & Deficiente \\
\hline 25 & 4”77 & Buena & $4 " 70$ & Buena & 51 & $5 \% 14$ & Buena & $5 \% 00$ & Buena \\
\hline
\end{tabular}

Fuente: Encuesta a deportistas

Elaborado por: Usca Renán, 2016 
Tabla 10. Análisis del pre y post test de precisión de coordinación

\begin{tabular}{|l|c|c|c|}
\hline \multicolumn{1}{|c|}{ Categorías } & Intervalos & Porcentajes PRE & Porcentajes POST \\
\hline EXCELENTE & 5 & $10 \%$ & $12 \%$ \\
\hline MUY BUENA & 4 & $16 \%$ & $32 \%$ \\
\hline BUENA & 3 & $28 \%$ & $34 \%$ \\
\hline REGULAR & 2 & $36 \%$ & $18 \%$ \\
\hline DEFICIENTE & $1-0$ & $10 \%$ & $4 \%$ \\
\hline \multicolumn{2}{r}{ TOTAL } & $\mathbf{1 0 0 \%}$ & $\mathbf{1 0 0 \%}$ \\
\hline
\end{tabular}

Fuente: Análisis del pre y post test

Elaborado por: Usca Renán, 2016

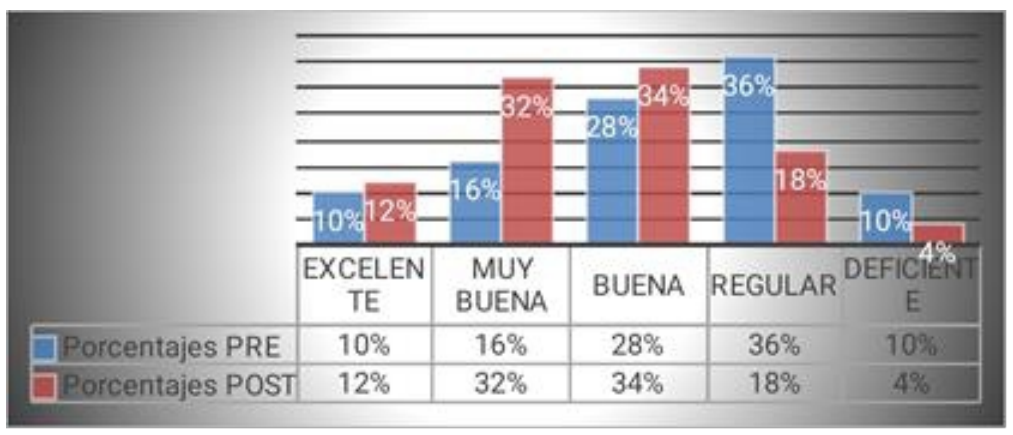

Gráfico 9. Pre y post test de coordinación

Análisis: En base al cuadro estadístico, podemos observar que mediante este programa para mejorar la coordinación y precisión de golpe de balón borde interno en las deportistas de la Academia de Fútbol Femenino Innovagol, se logró el objetivo requerido que era el mejoramiento de esta capacidad.

Interpretación: De acuerdo al análisis gráfico se ve que la mayoría de las deportistas de la Academia de Fútbol Femenino Innovagol ha mejorado favorablemente y estos valores se ven reflejados en este cuadro ya que podemos observar que en el pre existen valores muy bajos y gracias al entrenamiento en el post test se ve una mejoría, por ende en el cuadro podemos observar valores ya altos.

Tabla 11. Pre test de coordinación

\begin{tabular}{|l|c|c|c|}
\hline \multicolumn{1}{|c|}{ Categorías } & Intervalos & Frecuencias & Porcentajes \\
\hline EXCELENTE & $3 " 25-4 " 14$ & 5 & $10 \%$ \\
\hline MUY BUENA & 4 4"15-4"58 & 8 & $16 \%$ \\
\hline BUENA & $4 " 59-5 " 46$ & 14 & $28 \%$ \\
\hline REGULAR & $5 " 47-5 " 90$ & 18 & $36 \%$ \\
\hline DEFICIENTE & $5 " 91-6 " 35$ & 5 & $10 \%$ \\
\hline TOTAL & 50 & $100 \%$ \\
\hline
\end{tabular}

Fuente: Resultados Pre test de coordinación

Elaborado por: Usca Renán, 2016 


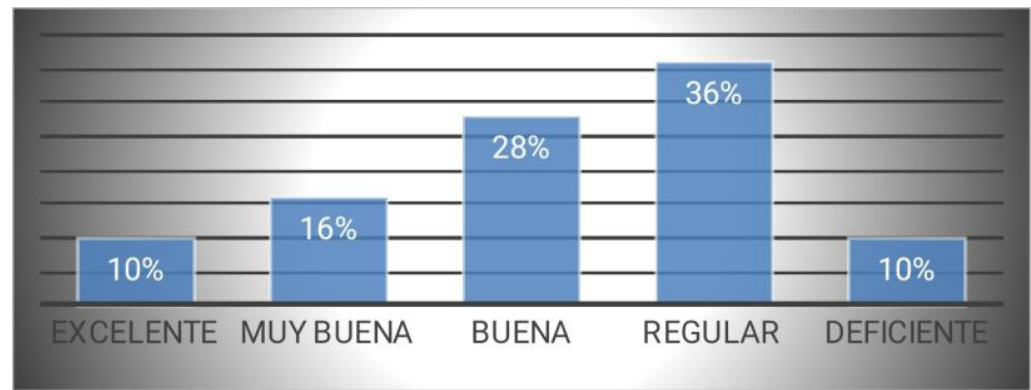

Gráfico 10. Test de coordinación

Análisis: Mediante el cuadro estadístico, el $10 \%$ de deportistas que realizaron el test se encuentran en el rango de excelente, el $16 \%$ de deportistas que realizaron el test se encuentran en el rango de muy buena, el $28 \%$ de deportistas que realizaron el test se encuentran en el rango de buena, el $36 \%$ de deportistas que realizaron el test se encuentran en el rango de regular, y el $10 \%$ de deportistas que realizaron el test se encuentran en el rango de deficiente.

Interpretación: En este cuadro estadístico observamos que las deportistas no tienen su $100 \%$ de deportistas con una buena coordinación, por esta razón trabajaremos esta capacidad constantemente para que haya una mejoría y así obtener nuestro objetivo propuesto.

Tabla 12. Post test de coordinación

\begin{tabular}{|c|c|c|c|c|c|}
\hline Categorías & \multicolumn{3}{|c|}{ Intervalos } & Frecuencias & Porcentajes \\
\hline EXCELENTE & 3"25 & - & 4"14 & 6 & $12 \%$ \\
\hline MUY BUENA & $4 " 15$ & - & $4 " 58$ & 8 & $16 \%$ \\
\hline BUENA & $4 " 59$ & - & $5 " 46$ & 13 & $26 \%$ \\
\hline REGULAR & $5 " 47$ & - & $5 " 90$ & 18 & $36 \%$ \\
\hline \multirow[t]{2}{*}{ DEFICIENTE } & $5 " 91$ & - & $6 " 35$ & 5 & $10 \%$ \\
\hline & \multicolumn{3}{|c|}{ TOTAL } & 50 & $100 \%$ \\
\hline
\end{tabular}

Fuente: Resultados Post test de coordinación

Elaborado por: Usca Renán, 2016

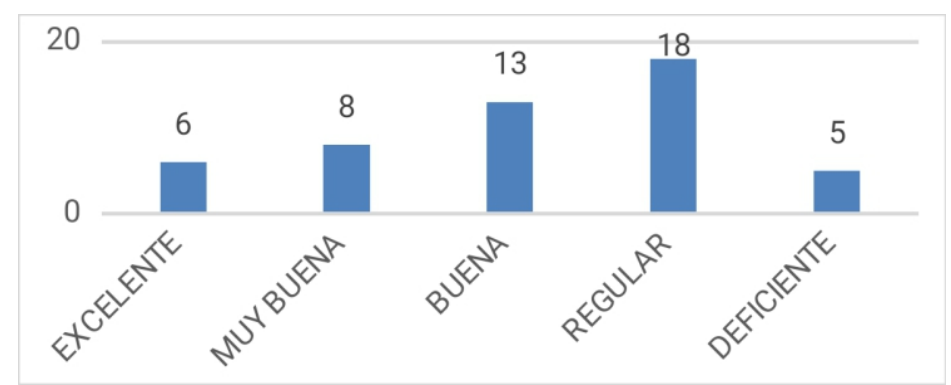

Gráfico 11. Post test de coordinación 
Análisis: El cuadro estadístico muestra que el $12 \%$ de las deportistas que realizaron el test se encuentran en el rango de excelente, el $16 \%$ se encuentra en el rango de muy buena, el $26 \%$ de las deportistas que realizaron el test se encuentran en el rango de buena, el $36 \%$ de las deportistas que realizaron el test se encuentran en el rango de regular, y el $10 \%$ se encuentra en el rango de deficiente.

Interpretación: En este cuadro estadístico observamos que existe una mejoría en comparación al primer test que se realizó, ya que por medio del trabajo de coordinación logramos mejorar a las deportistas en esta capacidad al realizar este segundo test.

\begin{tabular}{|r|c|c|c|c|}
\hline & \multicolumn{2}{|c|}{ Análisis estadístico Pre-test } & \multicolumn{2}{c|}{ Análisis estadístico Post-test } \\
\hline Criterio & Valor & Observación & Valor & Observación \\
\hline Media & 5,1456 & & 5,0030 & \\
\hline Moda & 5 "47-5"90 & Regular & 5 "47-5"90 & Regular \\
\hline Desviación estándar & 0,69837285 & & 0,69843470 & \\
\hline
\end{tabular}

Criterio T-Student: 0,8365

$\mathrm{P} \geq 0,05$

Los resultados no son significativos

\section{Conclusiones}

Luego de realizar el análisis en las tablas comparativas del pre test de coordinación encontramos que $74 \%$ de las deportistas están dentro de los rangos bueno, regular y deficiente y en el Post test de coordinación observamos que las deportistas se ubicaron en un rango de muy buena, con un tiempo dentro de 4"15 y 4"58 segundos; sin embargo, los valores no fueron significativos, por lo que se necesita incluir en el programa de intervención ejercicios para mejorar la precisión en el golpe del balón.

La encuesta permitió detectar que los entrenadores deben iniciar con un test de coordinación (escalera) y definir un programa de mejoramiento de la coordinación de golpe de balón en la categoría sub 18, pues se observó que en las deportistas vive el desconocimiento y poco interés para mejorar esta deficiencia.

Es de vital importancia que los entrenadores consideren la precisión en el golpe de balón con borde interno pues se detectaron problemas al ejecutarlo, y sin duda alguna se debe complementar con un nuevo programa de ejercicios conjuntamente con una evaluación, lo cual representa tema idóneo para ampliar una próxima investigación sobre este aspecto.

\section{References:}

1. López (2004). Eduardo: "Las Capacidades Coordinativas Gentileza". www.plazadedeportes.com/imgnoticias/7172.pdf Uruguay 
2. López \& Garoz (2004). Evaluación de las capacidades coordinativas, Barcelona, Graó

3. Lorenzo, F. (2002). Diseño y estudio científico de un test motor original que mida la coordinación motriz en alumnos/as de Educación Secundaria Obligatoria. Universidad de Granada. Tesis doctoral, no publicado.

4. Schreiner, P. (2005). Entrenamiento de la Coordinación en el Fútbol. Editorial Paidotribo.

5. Weineck, J. (2009). "Entrenamiento de la Coordinación en el Fútbol". Editorial Paidotribo. Barcelona, acceso: 16 de Octubre del. desacargadefutbol.blogspot.com/2009/10/entrenamiento-de-lacoordinacion-en- el_16.html

6. Weineck, J. (2005). Entrenamiento Total. Editorial Paidotribo. Barcelona.

7. Weineck, J. (1998). Entrenamiento óptimo, Barcelona, Editorial Hispano Europea. 